\title{
Editorial
}

This issue no. 2 of volume 3(2009) of the journal Advances in Data Analysis and Classification (ADAC) contains four articles that deal with the usage of string distance in phylogeny, with the selection of variables in model-based clustering, with clustering under the presence of outlying observations, and with Robinsonian dissimilarities.

- The first one, written by Fréderic Guyon, Céline Brochier-Armanet and Alain Guénoche, considers the problem of reconstructing phylogenies from the complete genomes of the underlying objects (beings). Typical algorithmic approaches are based on the computation of pairwise distances between the observed gene sequences (strings), and there are various options for measuring distance. This paper compares the performance of four string distances (all based on common word properties) when reconstructing phylogenetic trees (with a neighbour-joining strategy), by simulation experiments and for real data situations. In the former case, the data are obtained by random tree models that allow for base substitutions and fragment insertion/deletion. It is concluded that optimum results are obtained when computing distances from Maximum Significant Matches.

- Also the second article of this issue, written by Wilson Toussile and Elisabeth Gassiat, originates from a biological context, namely population genetics, where genetically homogeneous populations of individuals should be identified. Here the $L$-dimensional data vector of each individual comprizes its genotypes (categorical variables) at $L$ investigated loci, the same for each individual, and it is conjectured that an underlying clustering structure is based only on the genotypes from an (unknown) subset $S$ of all $L$ loci. The problem then consists in determining this subset $S$, the number of clusters, and the classification of the individuals. This problem is solved in the framework of a probabilistic model and leads to the optimization of penalized maximum likelihood criteria. The authors prove the consistency of their estimation method and propose a modified backward-stepwise algorithm for optimization. Experiments with simulated and real datasets underline the usefulness of the proposed loci selection procedure. 
- In the third article Maria Teresa Gallegos and Gunter Ritter propose a new method for clustering a set of data points in situations where outliers are to be expected and investigate analytically the robustness behaviour of the resulting clustering method. Their probabilistic clustering model starts from a trimmed likelihood function that does not involve the (a priori unknown) set of putative outliers and includes inequalities (HDBT constraints) for the class-specific covariance matrices that avoid degeneration in the heteroscedastic (normal) case. The proposed clustering algorithm proceeds by maximizing a trimmed a posteriori cluster criterion, or by minimizing a trimmed determinant criterion (TDC), and generalizes the classical $k$-means algorithm by a trimming step. By varying the number of putative outliers and the number of clusters, a list of interesting cluster/outlier configurations is proposed from which an optimum configuration is finally selected. A major merit of this paper is the fact that it determines the (restricted) breakdown points of the resulting clustering procedure and insofar characterizes the robustness properties of this approach. The results are illustrated by two examples.

- Finally, the article by Matthijs J. Warrens investigates if we can reconstruct an unknown order on a given set of objects if we have binary data for these objects (where an order may be characterized by consecutive ones in the data vectors), but want to use a clustering strategy that uses a dissimilarity matrix that is computed from the binary data. This problem is related to Robinsonian dissimilarity matrices where (after a suitable permutation of rows and columns, thereby creating an ordering of objects) dissimilarities increase when moving away from the main diagonal. The paper derives, for four dissimilarity measures $d$, sufficient conditions for $d$ being Robinsonian and insofar able to recover a hidden 'consecutive ones' structure of the underlying objects.

On its last pages this issue contains a Call for Papers for a Special Issue of ADAC devoted to the topic

\section{Network analysis}

Guest editors will be Anushka Ferligoj and Vladimir Batagelj. All ADAC readers are kindly encouraged to submit manuscripts for this issue. For details and deadlines see the Call for Papers and the ADAC website www.springer.com/11634.

Hans-Hermann Bock (Aachen)

Wolfgang Gaul (Karlsruhe)

Akinori Okada (Tokyo)

Maurizio Vichi (Rome) 